\title{
Characters of surface deformation and surface wave in thermal capillary convection
}

\author{
DUAN Li, KANG Qi \& HU Wenrei \\ National Microgravity Laboratory/CAS, Institute of Mechanics, Chinese Academy of Sciences, Beijing \\ 100080, China \\ Correspondence should be addressed to Duan Li (email: duanli@imech.ac.cn) \\ Received April 2, 2005; accepted June 27, 2006
}

\begin{abstract}
In the field of fluid mechanics, free surface phenomena is one of the most important physical processes. In the present research work, the surface deformation and surface wave caused by temperature difference of sidewalls in a rectangular cavity have been investigated. The horizontal cross-section of the container is $52 \mathrm{~mm} \times 42 \mathrm{~mm}$, and there is a silicon oil layer of height $3.5 \mathrm{~mm}$ in the experimental cavity. Temperature difference between the two side walls of the cavity is increased gradually, and the flow on the liquid layer will develop from stable convection to un-stable convection. An optical diagnostic system consisting of a modified Michelson interferometer and image processor has been developed for study of the surface deformation and surface wave of thermal capillary convection. The Fourier transformation method is used to interferometer fringe analysis. The quantitative results of surface deformation and surface wave have been calculated from a serial of the interference fringe patterns. The characters of surface deformation and surface wave have been obtained. They are related with temperature gradient and surface tension. Surface deformation is fluctuant with time, which shows the character of surface wave. The cycle period of the wave is $4.8 \mathrm{~s}$, and the amplitudes are from 0 to $0.55 \mu \mathrm{m}$. The phase of the wave near the cool side of the cavity is opposite and correlative to that near the hot side. The present experiment proves that the surface wave of thermal capillary convection exists on liquid free surface, and it is wrapped in surface deformation.
\end{abstract}

Keywords: surface deformation, surface wave, thermocapillary convection, buoyancy convection, optical interferometer.

\section{Introduction}

The thermal capillary convection is driven by non-uniformity of surface tension, which comes from non-uniformity of surface temperature. It is important fluid convec- 
tion phenomena in many fields, such as crystal growth and film science. All these physical phenomena are very important to influence many physics processes. Exploring them is available in controlling materials growing, and obtaining the advanced materials.

The convection brings on the surface flow of fluid, and forms a return-flow because of mass conservation. In mechanics theories, there is a shear flow near the free surface, and it is easy to cause instability or oscillation. When thermal capillary convection occurs, surface deformation and surface wave exist on the fluid surface. It is one of the essential characters in the process of flow translating. It is important to study them for understanding the mechanism of the kind of fluid convection. By now, studying surface deformation and surface wave are mainly focused on the theory field ${ }^{[1-3]}$. Sen and Davis ${ }^{[3]}$ prove that the pressure gradient is directed from the hot side towards the cold side, hence the pressure is higher at the cold side, and the interface bulges near the cold side and is constricted near the hot side. Our experiment results are the same as their theory results. While studying the mechanism of the convection oscillation in theories, one method is that the hydrothermal wave is obtained in the case of ametabolic free surface. Another method is that the surface wave is obtained in the case of transformative free surface. The hydrothermal wave has been observed clearly, and the hydrothermal wave instability also has been discussed and analyzed ${ }^{[4,5]}$. However, surface deformation is usually present if convection occurs, and the surface wave will possibly appear on free surface while convection transients into an oscillatory convection. Dabiri ${ }^{[6]}$ measured free surface deformation caused by near-surface turbulence, and he thought that there was storing energy in surface deformation; when the deformation relaxes, energy is released back into fluid, thereby affects the turbulence. It is important to study clearly surface deformation and surface wave. In recent years, surface waves in a floating half zone bridge have been measured by optical methods ${ }^{[7,8]}$, and analyzed in theories ${ }^{[1,2]}$.

While discussing an actual system of thermal capillary convection, thermal transfer is important. The coupling of thermal transfer and convection driven by the gradient of surface tension is the basic character. In the present research work, the technique of optical interference has been introduced for the measurement of fluid free surface deformation in microns order. The optical diagnostic system consisting of Michelson interferometer increases the measuring sensitivity in comparison with the conventional experimental method in fluid mechanics. And the image processor has been developed for obtaining quantitative results. The optical interferometer has been proved to be a good measurement method to study micro surface deformation of fluid. The present experiment also demonstrates that surface deformation and surface wave are very significative physics phenomena on thermal capillary convection. It is related with the convection introduced by surface tension gradient, and also related with temperature gradient in fluid surface. The interesting experiment results are given in the present paper.

\section{The experiment model and the diagnostics method}

In the present research work, the surface deformation and surface wave of the thermal capillary convection in a rectangular cavity are studied. The size of horizontal 
cross-section of the cavity is $52 \mathrm{~mm} \times 42 \mathrm{~mm}$. The two opposite lateral walls are made of transparent $\mathrm{K} 9$ glass for flow visualization. Other two opposite lateral walls are made of copperplates. There is a silicon oil layer in the experimental cavity. One of the copperplates is heated by an electro-thermal film, and the other one is cooled by a Peltier element with a radiator. The temperature difference between the two copperplates on the liquid layer will form, which will be measured by a T type thermocouple, and the hot side is controlled by a EUROTHERM temperature controller and DC power source. The bottom of the cavity is made from adiabatic materials, and the light reflecting capability of its surface is very poor. In the present experiment, the temperature difference is increased gradually, and the flow in liquid layer will develop from stable convection to un-stable convection. The optical interference method has been used to measure surface deformation and surface wave, in order to analyze the relation and the mechanism between the convection and surface deformation.

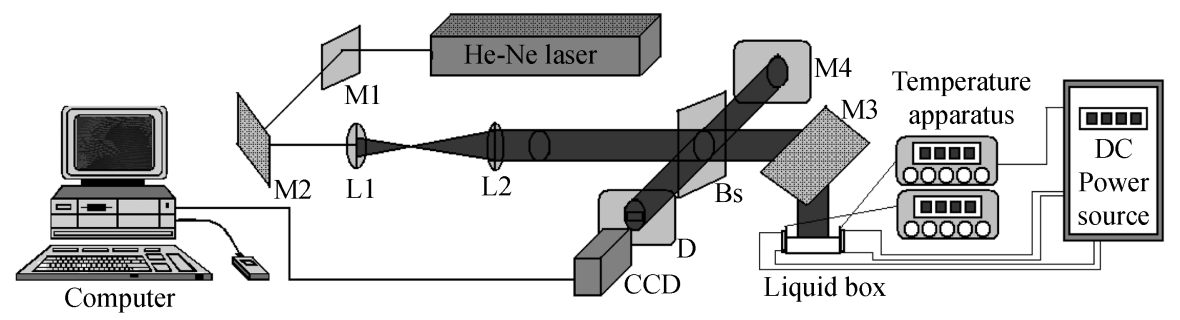

Fig. 1. Optical interference system.

In the present experiment, the Michelson interference method has been used to measure surface deformation of fluid in microns order. The diagram of the optical diagnostic system is shown in Fig. 1. A He-Ne laser beam passes through lens L1 and L2 to form an expanded parallel light beam, and then the beam is splitted by beam splitter Bs into two parallel light beams. One is used as the object beam, and the other is used as reference beam. The object light beam illuminates liquid surface, and is reflected from the surface, then is reflected again by beam splitter Bs. The reference light beam is reflected by mirror M4, and then passes through beam splitter Bs. At this time, the object beam and reference beam meet together and form an interferometric fringes pattern on the ground glass D. The fringes carry out information of surface deformation caused by capillary convection. The area measured by the interferometer is $18 \mathrm{~mm} \times 14.4 \mathrm{~mm}$, which is at the center of the cavity for the reason of horizontal liquid surface. The image system is composed by a CCD camera and Pinnacle image system in computer.

The characteristics of the measurement system are that the surface deformations in the whole view field can be synchronously obtained and the evolution of interference fringe image is also recorded at the rate of $25 \mathrm{frame} / \mathrm{s}$ in real-time, which reflect the free surface shape information at different temperature gradients. The precision of the spatial and temporal measurement is good enough to our requirement. Because, the vertical surface deformation caused by motion of one fringe is half wave length, and the precision of image processing is better than $1 / 10$ fringe. Therefore, the precision of the system can reach 
$\lambda / 20=0.032 \mu \mathrm{m}$ (Laser wave length $\lambda=0.6328 \mu \mathrm{m}$ ) at least. In the present experiment, the surface deformation is about several decades' microns, and the amplitude of surface wave is close to $1 \mu \mathrm{m}$. In addition, the period of surface wave is about $4.8 \mathrm{~s}$, however, the capturing period of video image is $0.040 \mathrm{~s}$, and the relative error of time measurement is less than $1 \%$. So, this optical interference system is promising for our research work.

In order to calculate surface deformation from interference fringes, grating analysis with the help of Fourier transform has been used. The fringe image at temperature difference $0^{\circ} \mathrm{C}$ is used as the original grating, and the fringe image at another state is used as the metamorphic grating. First, the phase distributions $\phi_{2}$ and $\phi_{1}$ of the metamorphic grating and the original grating are obtained by Fourier transformation of their fringe images respectively. Thus, we can work out the phase difference $\Delta \phi=\phi_{2}-\phi_{1}$. Finally, the vertical surface deformation of fluid surface $\Delta Z(x, y)$ can be written as follows:

$$
\Delta Z(x, y)=\frac{1}{2 n} \times \Delta \phi(x, y) \times \frac{\lambda}{2 \pi},
$$

where $n$ is the refractive index of air.

\section{Experimental result}

In the present experiment, the surface deformation of silicon oil layers in the height of liquid layer $h=3.5 \mathrm{~mm}$ has been observed. The temperature difference between the two sides of the cavity is increased from $0^{\circ} \mathrm{C}$ to $58.5^{\circ} \mathrm{C}$ at the rate of $0.73^{\circ} \mathrm{C} / \mathrm{min}$. The optical interference method measures surface deformation and surface wave of the convection. The interference fringes at temperature difference of $0^{\circ} \mathrm{C}$ is modulated to be horizontal (shown in Fig. 2), which is to be as the original fringe for the late calculation. The metamorphic fringe images, which stand for the deformation of liquid free surface, have been captured.

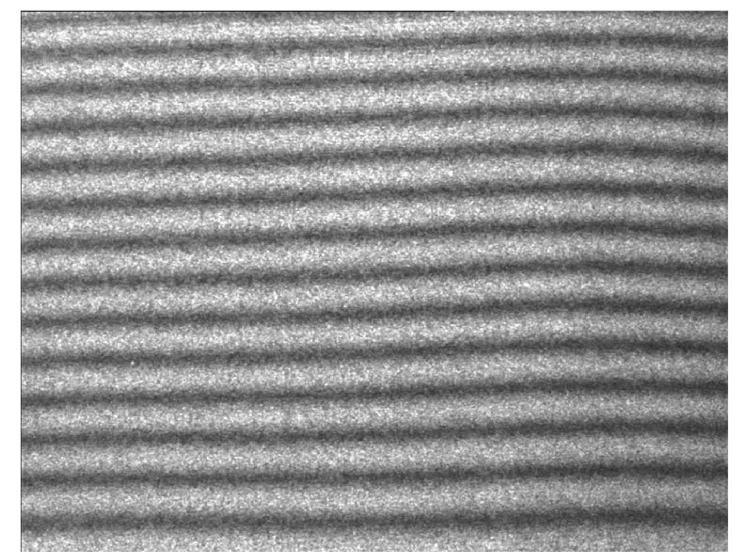

Fig. 2. The interference fringes when temperature difference $\Delta T=0.0^{\circ} \mathrm{C}$.

Fig. 3 shows the evolution of the interference fringes with the increasing of temperature difference. The bent fringe demonstrates that the liquid surface is more and more slanting with the increasing of temperature gradient. According to these interference 
fringe images, surface deformations have been calculated. The changes of deformation are from several microns to 50 microns, as shown in Fig. 4. In the figures, the direction of $X$-axis is the one of temperature gradient of fluid surface, and the direction of $Z$-axis is upward and vertical to level. The coordinate origin is at the center of fluid surface. The surface deformations of fluid are correlative directly with temperature gradient. It is a significant character on thermal capillary convection.
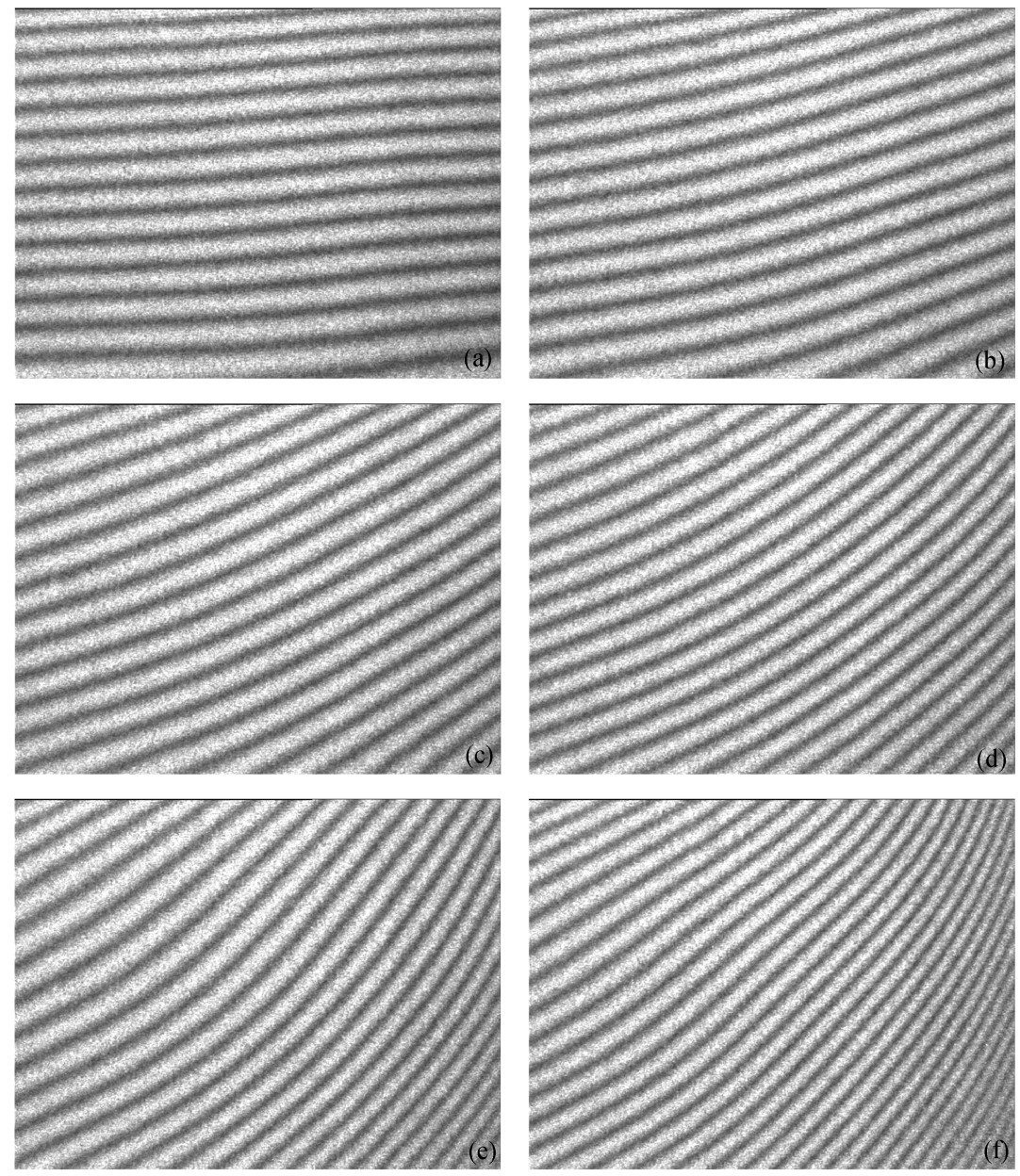

Fig. 3. Evolution of interference fringe images with temperature difference. (a) $\Delta T=6.0^{\circ} \mathrm{C}$; (b) $\Delta T=14.0^{\circ} \mathrm{C}$; (c) $\Delta T=22.0^{\circ} \mathrm{C}$; (d) $\Delta T=32.5^{\circ} \mathrm{C}$; (e) $\Delta T=42.5^{\circ} \mathrm{C}$; (f) $\Delta T=52.0^{\circ} \mathrm{C}$.

In order to show clearly the evolution process, the deformation on the central line ( $y=$ 0 ) is given out, as shown in Fig. 5. The experiment results demonstrate that surface deformation is related directly with temperature gradient. The larger the temperature gradient is, the more the surface deformation has. And the result also expresses that the cold side is higher than the hot side. From the experimental result of velocity field, surface' liquid is pulled from the hot side to the cold side ${ }^{[9]}$. It means that the driving force of convection is the surface tension. These experiment results are coincident with Sen and 


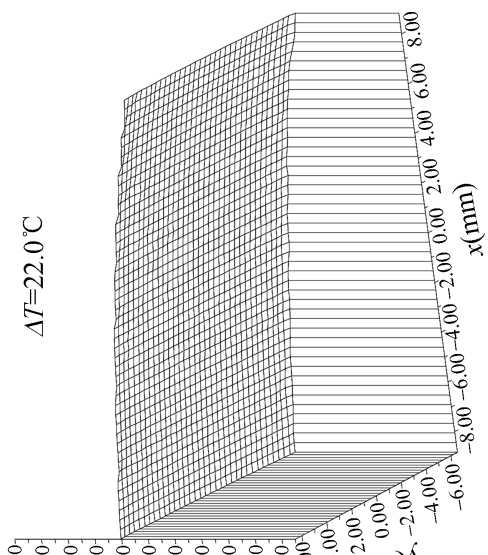

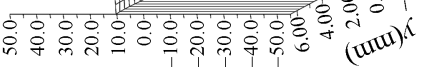
(uin) $z$

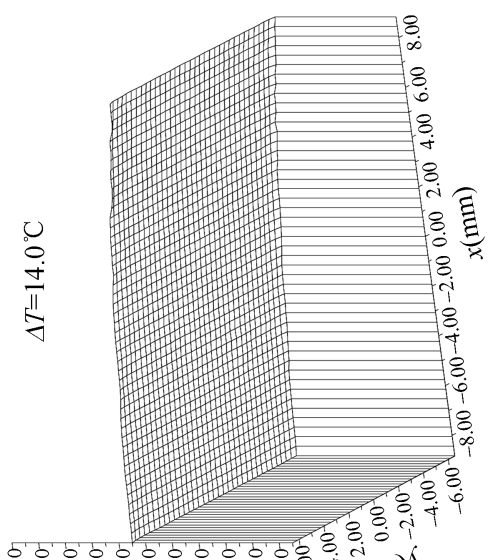

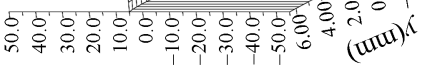
(uil) $z$

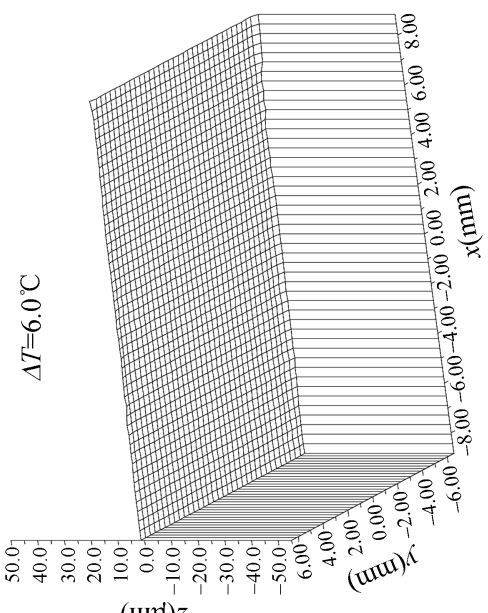

(uiv) $z$

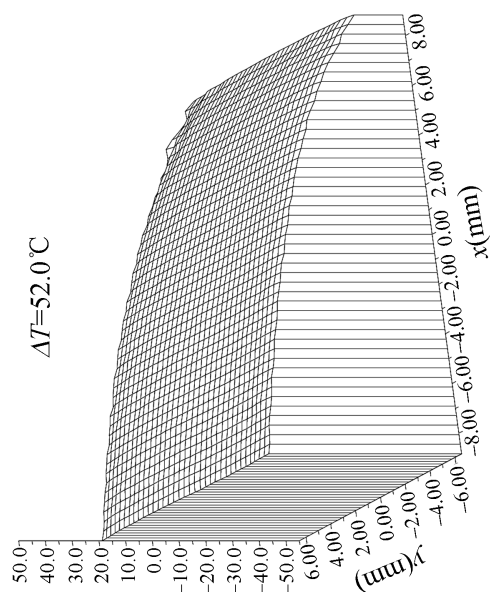

(uri) $z$

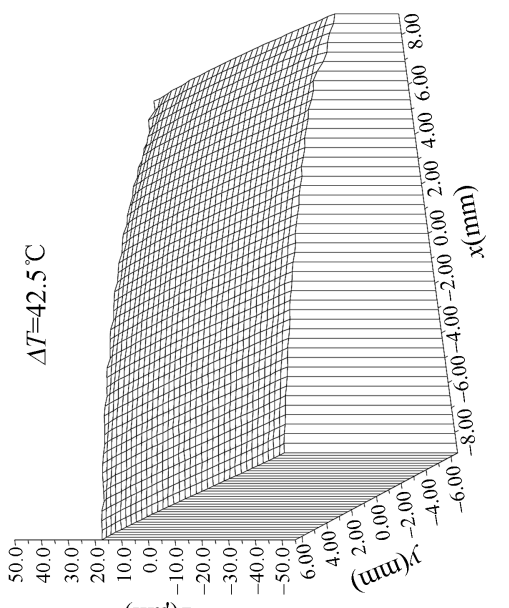

(uiv) $z$

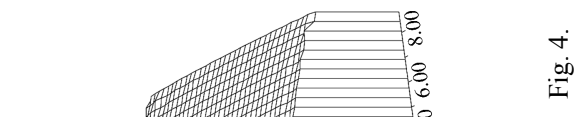

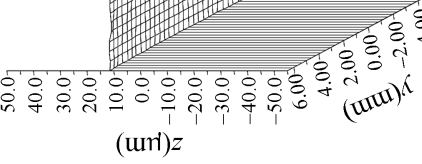

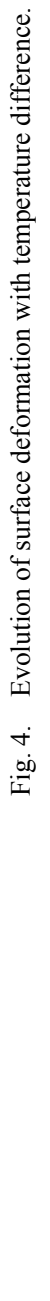


Davis's theory results ${ }^{[3]}$.

With temperature difference increasing, the phenomena of surface wave appear in the present experiment. They are often wrapped in surface deformation, because it is too slender to be observed. Its amplitude is generally less than one micron. In fact, when the temperature difference is large enough, the interference fringes curl at the hot side firstly, and then this curl fringes move to the cold side gradually. It demonstrates that surface wave exists on liquid surface.

In order to show the surface oscillation and surface wave clearly, the data of seven points on the liquid surface at temperature difference $32.5^{\circ} \mathrm{C}$ are fetched in a series time. The coordinate of the seven points are $(-7.3 \mathrm{~mm}, 0),(-5.0 \mathrm{~mm}, 0),(-2.7 \mathrm{~mm}, 0),(-0.4$ $\mathrm{mm}, 0),(1.9 \mathrm{~mm}, 0),(4.2 \mathrm{~mm}, 0)$ and $(6.5 \mathrm{~mm}, 0)$ respectively. Thus, the curves that the surface deformation changes with time are obtained, as shown in Fig. 6. Each curve stands for a different point on the central line of liquid surface. We discover that the sur-

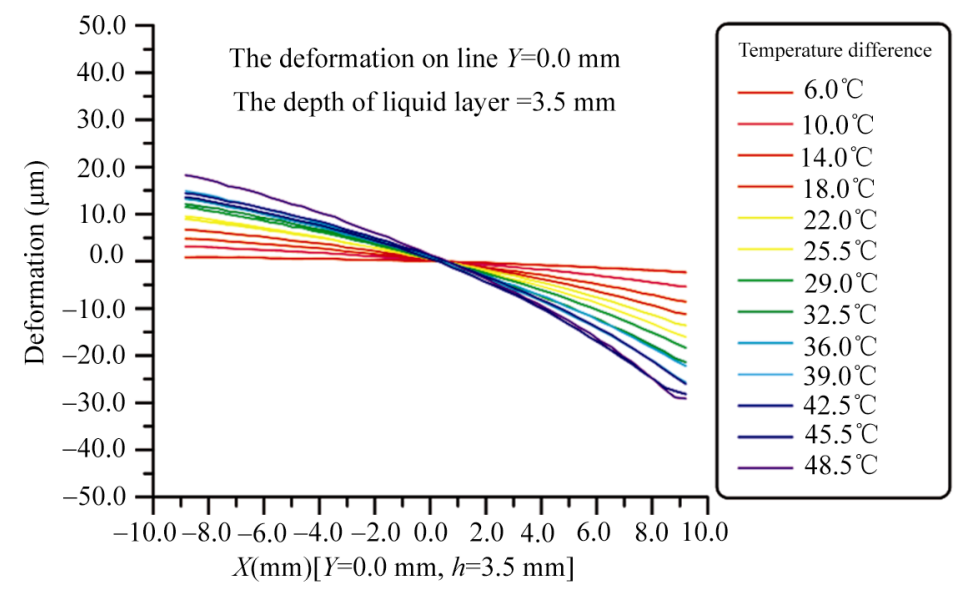

Fig. 5. Relationship of surface deformation with liquid thickness and temperature difference.

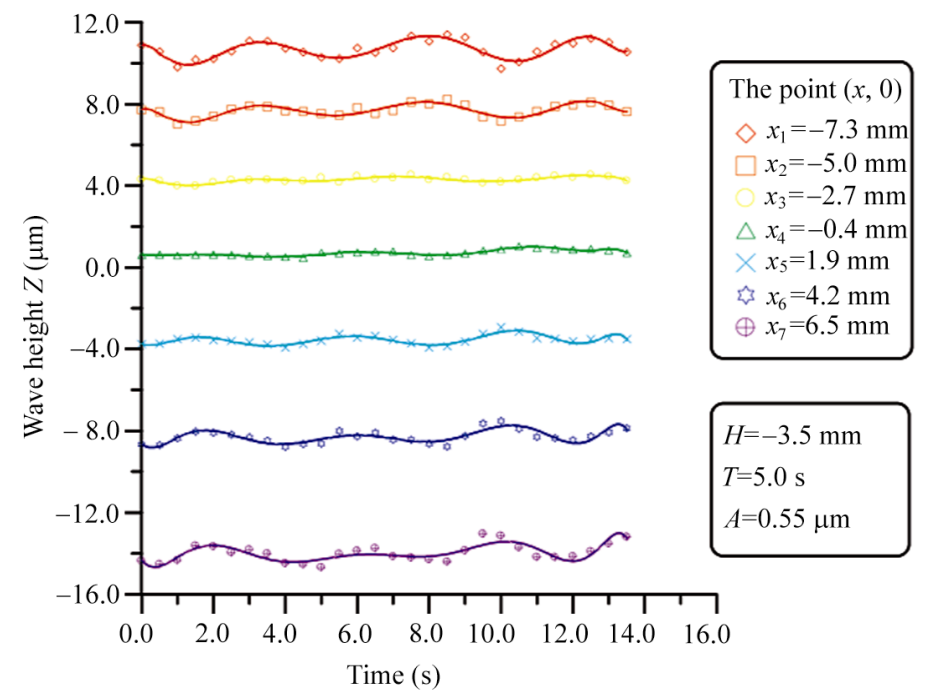

Fig. 6. The fluctuant phenomena of surface deformation with time. 
face deformation is fluctuant with time. It shows the characteristic of surface wave, and the cycle period is $4.8 \mathrm{~s}$, the amplitudes are about from 0 to $0.55 \mu \mathrm{m}$. Fig. 6 also demonstrates that the cycle periods for different points are equal, but the amplitudes are different; the amplitudes of the points on the hot side and the cold side are larger than that of the central points. The present experiment proves that surface wave of thermal capillary convection exists on liquid free surface.

This experiment was carried out on the ground. The effect of buoyancy force is generally ignored completely. The effect of the relative strength of buoyancy force to thermal capillary force on convection can be usually quantified in terms of the dynamic Bond number $B o$,

$$
B o=\frac{\rho g \beta d^{2}}{\gamma} .
$$

Here $\rho$ is density of fluid, $g$ is gravitational acceleration, $\beta$ is the thermal expansion coefficient, $\gamma$ is the negative rate of change of surface tension with temperature, and $d$ is the height of liquid layer. The surface tensions of $1000 \mathrm{cSt}$ silicon oil at different temperatures are measured by a Processor Tensiometer-K12 instrument, and are shown in Table 1 and Fig. 7. The surface tension $\sigma$ is proportional to temperature as the formula:

$$
\sigma=-0.0684438 \mathrm{~T}+24.8387 \text {. }
$$

Thus, the negative rate of change of surface tension with temperature $\gamma=d \sigma / d T=$ $-0.0684(\mathrm{dy} / \mathrm{cm} \cdot \mathrm{K})$.

Table 1 The surface tensions at different temperatures

\begin{tabular}{ccccccccccc}
\hline$T(\mathrm{~K})$ & 296.4 & 296.6 & 301.2 & 306.6 & 311.0 & 317.3 & 323.5 & 330.1 & 335.8 & 343.2 \\
\hline$\sigma(\mathrm{dy} / \mathrm{cm})$ & 23.27 & 23.32 & 22.93 & 22.52 & 22.15 & 21.69 & 21.37 & 20.88 & 20.61 & 20.10 \\
\hline
\end{tabular}

The Densimeter is used to measure the density $\rho$ of the silicon oil $1000 \mathrm{cSt}$ at different temperatures, shown in Table 2 and Fig. 8. The average value of the density is $\rho=0.9535$ $\mathrm{g} / \mathrm{cm}^{3}$, and the change rate of the density with temperature is $d \rho / d T=$ $-0.8713 \times 10^{-3}\left(\mathrm{~g} / \mathrm{cm}^{3}\right)$. Therefore, the thermal expansion coefficient $\beta$ can be written as follows:

Table 2 The densities in different temperatures

\begin{tabular}{cccccccccc}
\hline$T(\mathrm{~K})$ & 297.6 & 299.8 & 304.7 & 309.1 & 315.0 & 320.1 & 327.1 & 332.8 & 340.0 \\
\hline$\rho\left(\mathrm{g} / \mathrm{cm}^{3}\right)$ & 0.9705 & 0.9673 & 0.9630 & 0.9600 & 0.9540 & 0.9510 & 0.9430 & 0.9400 & 0.9325 \\
\hline
\end{tabular}

$$
\beta=\frac{d V}{V \cdot d T}=-\frac{d \rho}{\rho \cdot d T}=\frac{0.8713 \times 10^{-3}}{0.9535}=0.9138 \times 10^{-3}\left(\mathrm{~K}^{-1}\right)
$$

So, the Bond number of the experimental system is 1.53 for the height of the liquid layer $h=3.5 \mathrm{~mm}$. Because it is small, we can think that surface tension controls the convection. The driving force for thermal capillary convection is the gradient of temperature along horizontal direction on free surface of liquid. For the present convection, the fluid near free surface flows from the hot side to the cold side, and then turns back to the hot side 


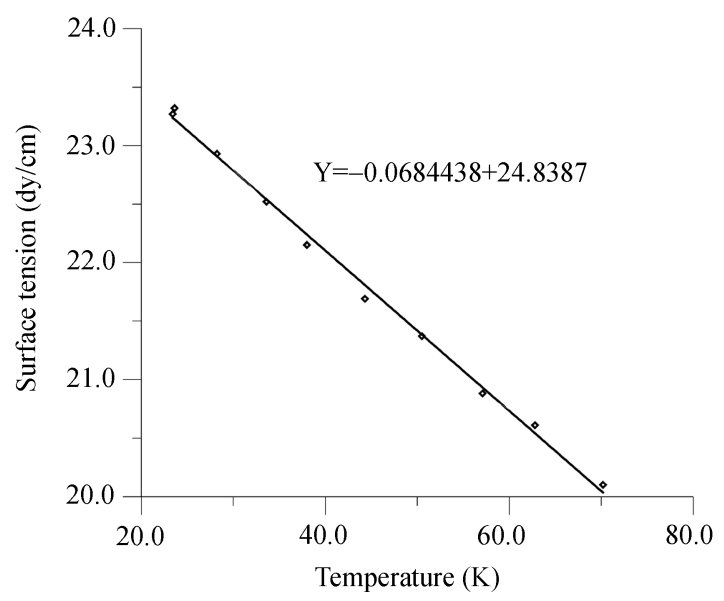

Fig. 7. Relationship between surface tension and different temperature.

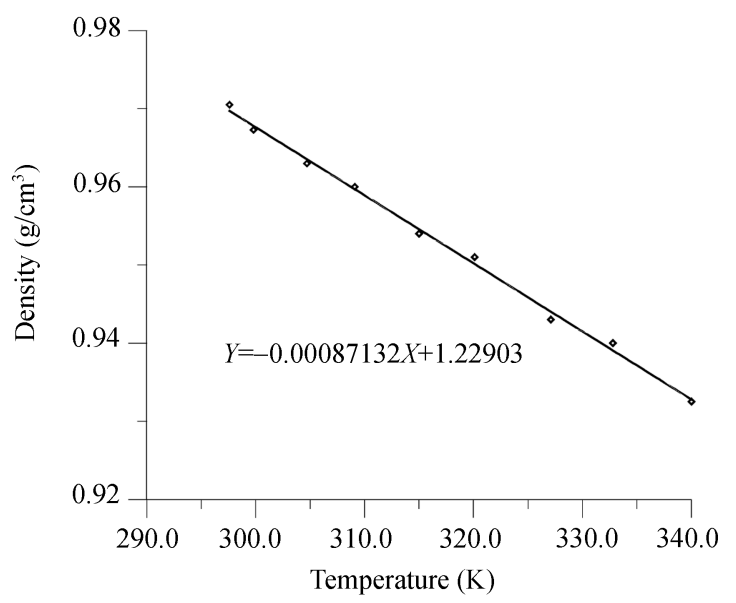

Fig. 8. Relationship between density $\rho$ and different temperature.

on the lower liquid layer ${ }^{[9]}$. So the temperature in the upper part of liquid layer is higher than that in the lower part of liquid layer, and the direction of temperature gradient in vertical direction is opposite to the direction of gravitational acceleration. Therefore, the fluid is stability in the buoyancy system. Though the gravity effect cannot be neglected, the convection is mainly dominated by thermal capillary force when the height of liquid layer is smaller.

\section{Conclusion}

An optical diagnostic system consisting of a modified Michelson interferometer and image processor has been developed for study of the kinetics of thermal capillary convection and buoyancy convection. This optical interferometer has been used to observe and measure surface deformation and surface wave of the thermal capillary convection in a rectangular cavity with different temperature's sidewalls. Fourier transformation is used 
in interference fringe image processing. The quantitative results of surface deformation and surface wave have been obtained from the interference fringe images. With the increasing of temperature gradient, the liquid surface slants gradually. The present experiment proves that the surface deformation appears before the appearance of surface wave in fluid convection, and it is related with temperature gradient. Surface deformation is fluctuant with time, which shows the characteristics of surface wave. The present experiment proves that surface wave of thermal capillary convection exists on liquid free surface, and it is wrapped in surface deformation. Besides, the dynamic Bond number $B o$ has been calculated in order to analyze the effect of buoyancy convection.

Acknowledgements This work was supported by the National Natural Science Foundation of China (Grant No. 10432060), and the Knowledge Innovation Program of the Chinese Academy of Sciences (Grant Nos. KJCX2-SW-L05, KSCX2-SW-322).

\section{References}

1 Hamed M, Floryan J M. Marangoni convection. Part 1. A cavity with differentially heated sidewalls. J Fluids, 2000, 405: 79-110

2 Smith M K, Davis S H. Instabilities of dynamic thermocapillary liquid layers. J Fluid Mech, 1983, 132: 145162

3 Sen A K, Davis S H. Steady thermocapillary flows in two-dimensional slots. J Fluid Mech, 1982, 121: 163186

4 Riley R J, Neitzel G P. Instability of thermocapillary-buoyancy convection in shallow layers. Part 1. Characterization of steady and oscillatory instabilities. J Fluid Mech, 1998, 359: 143-164

5 Burguete J, Mukolobwiez N, Daviaud F, et al. Chiffaudel, Buoyant-thermocapillary instabilities in extended liquid layers subjected to a horizontal temperature gradient. Physics of Fluids, 2001, 13(10): 2773 - 2787

6 Dabiri D, Gharib M. Simultaneous free-surface deformation and near-surface velocity measurements. Experiments in Fluids, 2001, 30: 381-390

$7 \mathrm{Hu} \mathrm{W} \mathrm{R,} \mathrm{You} \mathrm{H} \mathrm{T,} \mathrm{Cao} \mathrm{Z} \mathrm{H.} \mathrm{Free} \mathrm{surface} \mathrm{oscillation} \mathrm{of} \mathrm{thermalcapillary} \mathrm{convection} \mathrm{in} \mathrm{liquid} \mathrm{bridge} \mathrm{of} \mathrm{half}$ floating zone. Sci China Ser A, 1992, 35(9): 1101-1109.

8 Shu J Z, Yao Y L, Zhou H, et al. Experimental study of free surface oscillation of a liquid bridge by optical diagnostics. Microgravity Science and Technology, 1994, 7: 83

9 Kang Q, Duan L, Hu W R, Experimental study of surface deformation and flow pattern on Buoyant-thermocapillary convection. Microgravity Science \& Technology, 2004, 15(2): 18-24 